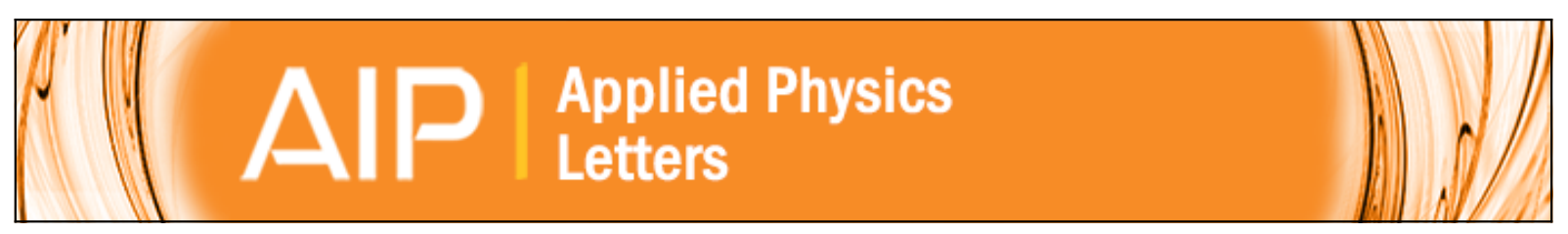

\title{
Double Dirac cones in phononic crystals
}

Yan Li, Ying Wu, and Jun Mei

Citation: Applied Physics Letters 105, 014107 (2014); doi: 10.1063/1.4890304

View online: http://dx.doi.org/10.1063/1.4890304

View Table of Contents: http://scitation.aip.org/content/aip/journal/apl/105/1?ver=pdfcov

Published by the AIP Publishing

\section{Articles you may be interested in}

Acoustic cloaking by a near-zero-index phononic crystal

Appl. Phys. Lett. 104, 161904 (2014); 10.1063/1.4873354

Love waves in two-dimensional phononic crystals with depth-dependent properties

Appl. Phys. Lett. 103, 111902 (2013); 10.1063/1.4820924

Laminated piezoelectric phononic crystal with imperfect interfaces

J. Appl. Phys. 111, 013505 (2012); 10.1063/1.3672404

Influences of gradient profile on the band gap of two-dimensional phononic crystal

J. Appl. Phys. 110, 103514 (2011); 10.1063/1.3660380

Lamb waves in phononic crystal slabs with square or rectangular symmetries

J. Appl. Phys. 104, 043506 (2008); 10.1063/1.2970067

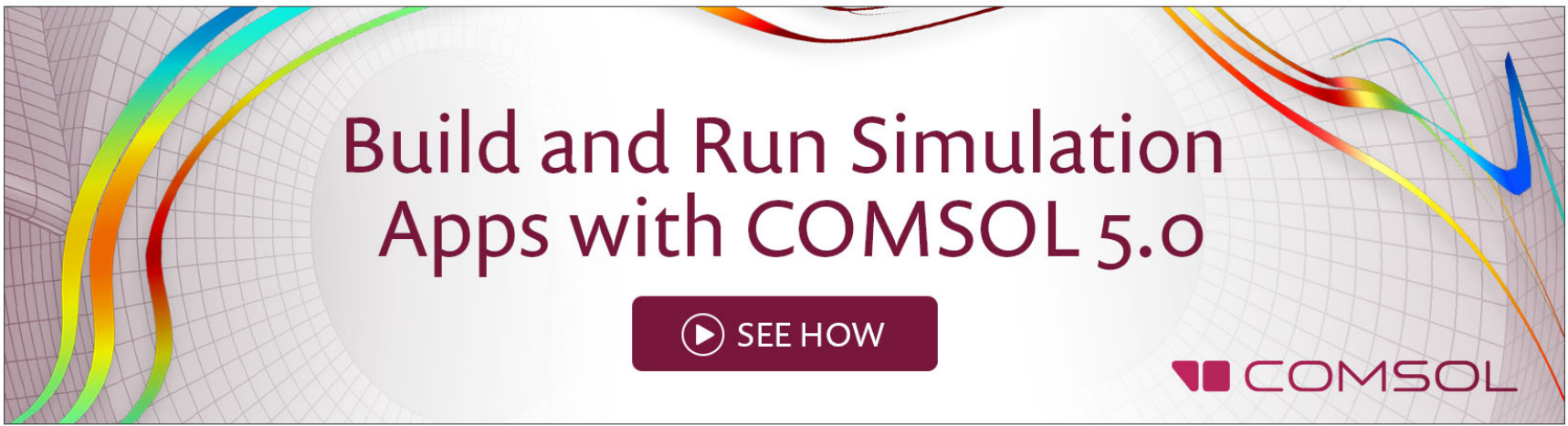




\title{
Double Dirac cones in phononic crystals
}

\author{
Yan $\mathrm{Li}^{1}{ }^{1}$ Ying Wu, ${ }^{2}$ and Jun $\mathrm{Mei}^{1, \mathrm{a})}$ \\ ${ }^{1}$ Department of Physics, South China University of Technology, Guangzhou 510640, China \\ ${ }^{2}$ Division of Computer, Electrical and Mathematical Sciences and Engineering, King Abdullah University \\ of Science and Technology (KAUST), Thuwal 23955-6900, Saudi Arabia
}

(Received 21 May 2014; accepted 3 July 2014; published online 11 July 2014)

\begin{abstract}
A double Dirac cone is realized at the center of the Brillouin zone of a two-dimensional phononic crystal (PC) consisting of a triangular array of core-shell-structure cylinders in water. The double Dirac cone is induced by the accidental degeneracy of two double-degenerate Bloch states. Using a perturbation method, we demonstrate that the double Dirac cone is composed of two identical and overlapping Dirac cones whose linear slopes can also be accurately predicted from the method. Because the double Dirac cone occurs at a relatively low frequency, a slab of the PC can be mapped onto a slab of zero refractive index material by using a standard retrieval method. Total transmission without phase change and energy tunneling at the double Dirac point frequency are unambiguously demonstrated by two examples. Potential applications can be expected in diverse fields such as acoustic wave manipulations and energy flow control. (C) 2014 AIP Publishing LLC.
\end{abstract}

[http://dx.doi.org/10.1063/1.4890304]

Many of graphene's interesting properties, including its integer quantum Hall effect, originate from its unique linear dispersion relations at the corner of the Brillouin zone (BZ), the so-called Dirac point. ${ }^{1}$ Not restricted to quantum systems, ${ }^{1-4}$ this special kind of linear dispersion relation is also found in classical periodic systems including photonic and phononic crystals (PCs), where interesting wave transport phenomena like pseudo-diffusion, cloaking of an object, and classical analogs of the Zitterbewegung have been studied. ${ }^{5-20}$ The linear dispersion relations in classical systems at high symmetry points in the BZ can be categorized into two types. ${ }^{20}$ One is located at the corner of the BZ, and the other is located at the center. The first type usually comes from a double-degenerate state, and there are two bands touching linearly at the Dirac point. The second type is called a Diraclike cone, which is a result of accidental degeneracy of a double-degenerate state and a single state. Thus, in addition to two bands touching linearly, there is a flat band intersecting the two linear bands at the Dirac-like point. Many studies of Dirac points in classical systems have been devoted to these two types of linear dispersion relations.

Recently, Sakoda ${ }^{21}$ predicted the existence of a different type of linear dispersion relation called the double Dirac cone in electromagnetic metamaterials and pointed out that the double Dirac point occurs at the BZ center, meaning that the effective refractive index is zero at that point. A double Dirac cone consists of a pair of two identical and overlapping Dirac cones. Thus, it requires a four-fold degenerate state at the double Dirac point, which makes the realization of a double Dirac cone in real systems extremely difficult. Until now, there has been only one report on realizing a double Dirac cone in an acoustic crystal with a honeycomb array of iron cylinders in water. ${ }^{22}$ That double Dirac cone was found when the wavelength was comparable to the lattice

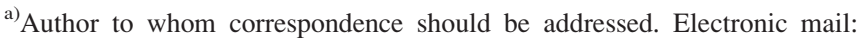
phjunmei@scut.edu.cn.
}

constant, deeming the effective medium description inappropriate.

In this work, we propose an acoustic system that possesses a double Dirac cone at a relatively low frequency. To understand the underlying physics and verify the dispersion of the double Dirac cone, we employ a perturbation method, which not only offers a link between the eigenstates and the linear dispersion but also accurately predicts the slopes of the dispersion relations. Different from the previously designed system, ${ }^{22}$ where the fields are concentrated in the host at resonances, the fields in our systems are concentrated in the scatterers, which lower the resonance frequency and make it possible to describe our system using effective medium theory. Around the double Dirac point frequency, we reveal that a slab of our acoustic system can be mapped onto an acoustic zero-index medium (ZIM) by using a standard retrieval method. ZIMs ${ }^{23-32}$ have spectacular applications, such as beam self-collimation, energy squeezing, and tunneling. Here, we demonstrate with two examples the interesting wave transport behaviors in our systems. These examples unambiguously show that zero phase change and total transmission of plane waves can be simultaneously achieved, both in a straight waveguide and in a U-shaped narrow neck waveguide channel.

The acoustic system considered in our work is a twodimensional (2D) PC consisting of a triangular array of coreshell cylinders embedded in a water host, as shown in the center of Fig. 1(a). The inner cores are iron rods with radii $R_{2}=0.2822 a$, and the shells are made of silicone rubber with outer radii $R_{1}=0.3497 a$, where $a$ is the lattice constant. The mass densities of water, rubber, and iron are $\rho_{0}=1000 \mathrm{~kg} / \mathrm{m}^{3}, \rho_{1}=1300 \mathrm{~kg} / \mathrm{m}^{3}$, and $\rho_{2}=7670 \mathrm{~kg} / \mathrm{m}^{3}$, respectively. The longitudinal wave velocities in water, rubber, and iron are $c_{0}=1490 \mathrm{~m} / \mathrm{s}, c_{1}=489.9 \mathrm{~m} / \mathrm{s}$, and $c_{2}=6010 \mathrm{~m} / \mathrm{s}$, respectively. We ignore the shear wave modes in the solid components in the simulation, as we will show later that this simplification does not alter the essential physics of the system. The band structures of the PC are 

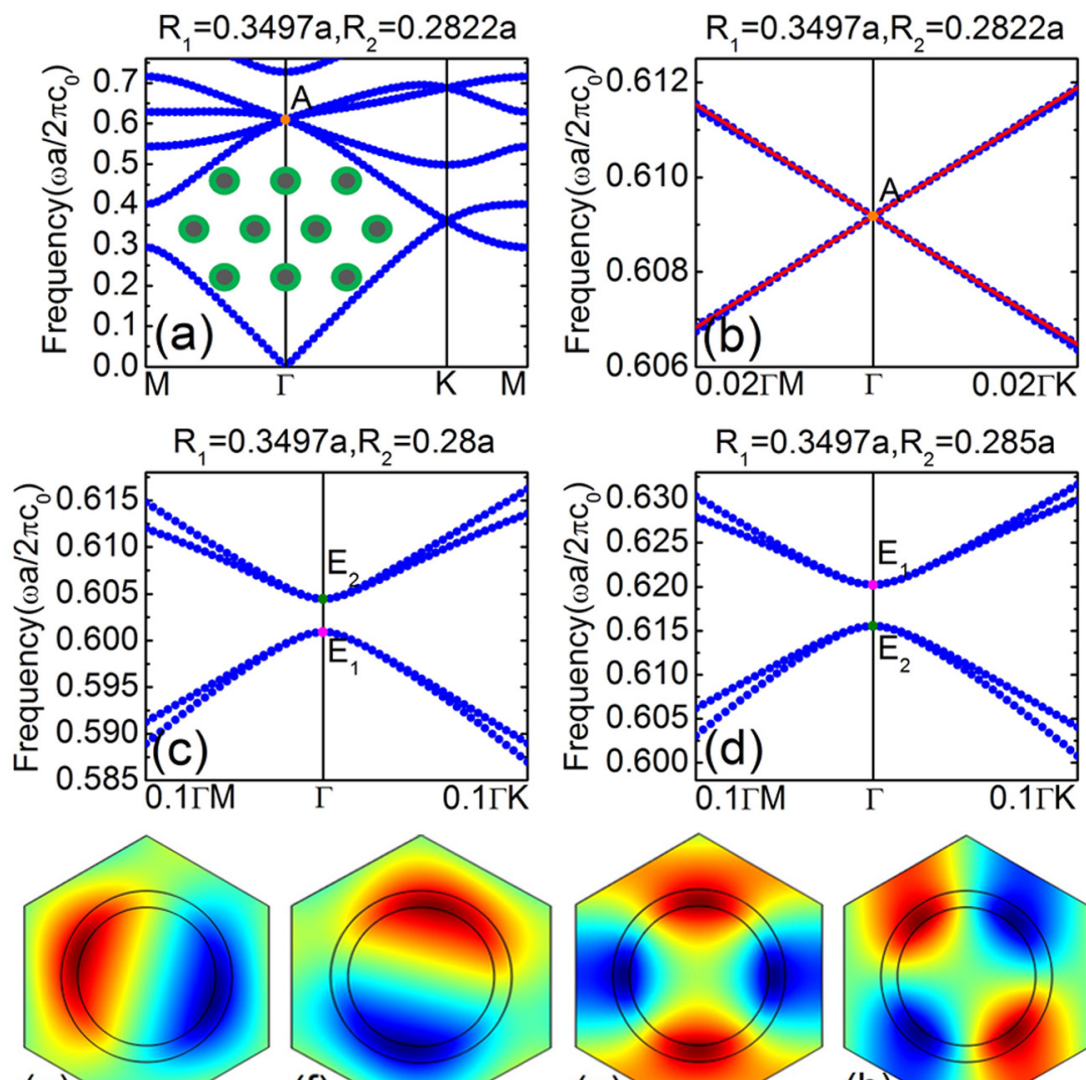

(e)
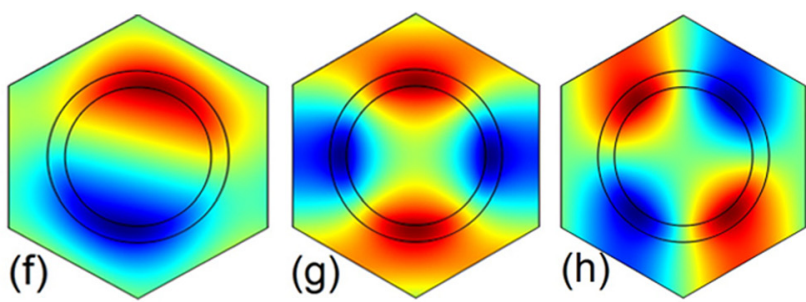

FIG. 1. Band structures of a twodimensional PC composed of a triangular array of rubber-coated iron cylinders embedded in a water host, where $R_{1}$ denotes the radii of the iron cores, and $R_{2}$ denotes the radii of the core + shell cylinders. The blue dots present the full-wave calculations obtained by using COMSOL Multiphysics. The red curves show the bands predicted by Eq. (2). (a) Band structures for $R_{1}=0.3497 a$ and $R_{2}=0.2822 a$, with $a$ being the lattice constant. Four-fold degeneracy at Point A is seen. (b) Enlarged view of (a) around Point A. (c) and (d) Band structures for different radii of iron cores, with $R_{2}=0.28 a$ in (c) and $R_{2}=$ $0.285 a$ in (d), where the four-fold degenerate state splits into two doubledegenerate states, marked as $E_{1}$ and $E_{2}$, respectively. (e)-(h) The pressure field distributions of the four degenerate eigenstates at Point $\mathrm{A}$, where dark red and dark blue denote the positive and negative maxima, respectively. The patterns in (e) and (f) are dipolar modes, and those in (g) and (h) are quadrupolar modes. calculated by using COMSOL Multiphysics, a commercial package based on the finite element method, and the results are plotted in Fig. 1(a), which exhibits a four-fold degenerate point, marked as "A", at the BZ center at frequency $\omega_{0}=0.6092\left(2 \pi c_{0} / a\right)$. In the vicinity of Point " $\mathrm{A}$ " the four bands collapse into two linear bands, as shown in Fig. 1(b), an enlarged view of the band structures near Point "A".

In Figs. 1(e)-1(h), we plot the pressure fields for the four degenerate Bloch states at Point "A", where a dipolar field pattern is seen in Figs. 1(e) and 1(f), and a quadrupolar pattern is recognized in Figs. 1(g) and 1(h). In a PC consisting of a triangular lattice of circular cylinders, the point group at the BZ center is $C_{6 v}$, which has two 2D irreducible representations: $E_{1}$ and $E_{2}$. In this case, the dipolar states correspond to $E_{1}$ representation, and the quadrupolar states correspond to $E_{2}$ representation. If we alter the inner and outer radii of the core-shell structure, the four-fold degeneracy will be lifted, and the dipolar states will be separated from the quadrupolar states, which are shown in Figs. 1(c) and 1(d). This indicates that the four-fold degeneracy at Point " $A$ " is a result of accidental degeneracy of a doubledegenerate dipolar state and a double-degenerate quadrupolar state.

The band structures shown in Fig. 1 are obtained without considering the shear modes in the solid components of the cylinders. As noted in Ref. 33, for a PC consisting of iron cylinders embedded in a water host, due to the large contrast between the longitudinal wave velocities of iron and water, the shear modes inside the solid cylinders can be safely ignored. Even though we have a layer of rubber coating around the iron in our PC system, we demonstrate in Fig. 2 that when the shear modes in the solid components are fully taken into account, the double Dirac cone still exists, and its main properties remain unchanged. Figure 2(a) shows the band structures around the $\Gamma$ point when the shear wave velocities are set to $c_{1 t}=200 \mathrm{~m} / \mathrm{s}$ for rubber and $c_{2 t}=$ $3231 \mathrm{~m} / \mathrm{s}$ for iron. The radii of the iron cores and the coated cylinders are chosen as $R_{2}=0.2696 a$ and $R_{1}=0.3416 a$, respectively. Despite of the slight changes in the geometric parameters and the resulting frequency, the existence of a double Dirac point is manifest in Fig. 2(a). The pressure field distributions for the eigenstates at the double Dirac point are plotted in Figs. 2(b)-2(e), which, indeed, exhibit similar behaviors as those shown in Figs. 1(e)-1(h). Both the band

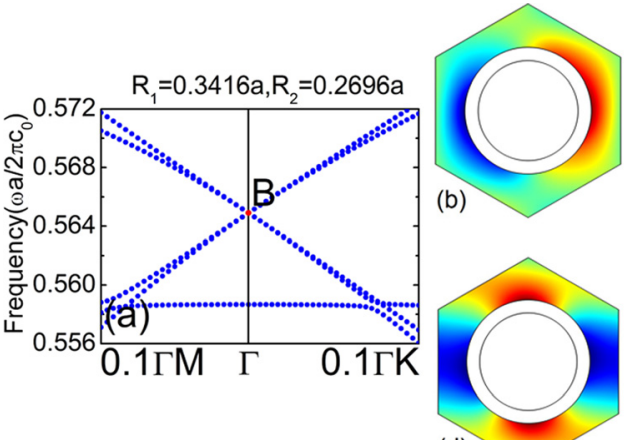

(d)
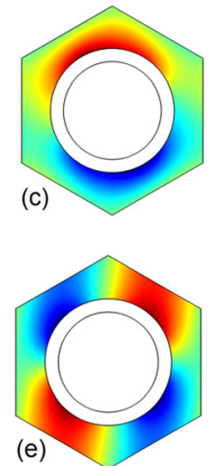

FIG. 2. Band structures and pressure field distributions for a $\mathrm{PC}$ that is nearly the same as the one shown in Fig. 1(b), except that the shear modes in iron and rubber are fully taken into account. (a) Band structures around the $\Gamma$ point, with $R_{2}=0.2696 a$ and $R_{1}=0.3416 a$. Four-fold degeneracy at Point B is seen. (b)-(e) Pressure field distributions in water for the four degenerate eigenstates at Point B. The pressure field patterns are very similar to those shown in Fig. 1, with (b) and (c) corresponding to the dipolar modes and (d) and (e) corresponding to the quadrupolar modes. 
structures and the field patterns of the eigenstates show that it is possible to neglect the shear mode. Thus, for simplicity but without losing the essential physics, we ignore the shear mode contributions in the following calculations.

The double Dirac cone possesses many interesting features. First, different from the previously studied Dirac-like cones at the BZ center, there is no flat band. Second, there seems to be only two bands touching linearly rather than four. Does this suggest that a double Dirac cone is composed of two identical Dirac cones or two different but very close Dirac cones? To answer this question and to gain deeper insight into the origin of the unusual linear dispersions, we adopt a perturbation method to study the double Dirac cone. The perturbation method ${ }^{18-20}$ is similar to the $\vec{k} \cdot \vec{p}$ method in quantum mechanics and can accurately predict the linear slopes of the bands. We assume that the four degenerate Bloch eigenstates shown in Figs. 1(e)-1(h) are known and denoted by $\varphi_{1}, \varphi_{2}, \varphi_{3}$, and $\varphi_{4}$, respectively. Taking these $\varphi_{i} \mathrm{~s}$ as the unperturbed basis to expand the Bloch eigenfunctions at a point near Point "A", we can then obtain the dispersion relations after solving the following secular equation (see Refs. 18-20 for details):

$$
\operatorname{det}\left|\begin{array}{cccc}
x+P_{11} & P_{12} & P_{13} & P_{14} \\
P_{21} & x+P_{22} & P_{23} & P_{24} \\
P_{31} & P_{32} & x+P_{33} & P_{34} \\
P_{41} & P_{42} & P_{43} & x+P_{44}
\end{array}\right|=0
$$

where $x=\left(\omega_{\vec{k}}^{2}-\omega_{0}^{2}\right) / c_{0}^{2}, \omega_{0}$ is the frequency of the double Dirac point, and $P_{l j}(l, j=1,2,3,4)$ represents the modecoupling integrals between the degenerate eigenstates, which may be calculated numerically. In the vicinity of Point " $\mathrm{A}$ ", where $\Delta k=\left|\vec{k}-\vec{k}_{0}\right|\left(\vec{k}_{0}=0\right.$ is the Bloch wave vector at Point "A"), the solution to Eq. (1), to the first order in $\Delta k$, can be written as

$$
\Delta \omega_{\vec{k}}=\omega_{\vec{k}}-\omega_{0}=\gamma_{\beta} \Delta k
$$

Two double roots, $\gamma_{\beta}= \pm 0.2037 c_{0}$, are found. These roots are the linear slopes of the bands predicted by the perturbation method. The results given by Eq. (2) are plotted as red curves in Fig. 1(b), while the results of the full-wave calculation obtained by using COMSOL Multiphysics are plotted as blue dots. They agree with each other very well, suggesting that the perturbation method is accurate. We emphasize the following two points. First, the double roots to Eq. (1) unambiguously suggest that there are two identical cones coinciding with each other and forming a double cone. Second, as predicted by Eq. (2), the linear slopes of the bands are exactly the same in all directions, meaning that the cone is isotropic.

In the vicinity of the double Dirac point, the Bloch wave vector, $\vec{k}$, is very small in magnitude, which means that, inside the PC, the accumulated phase change of the acoustic wave should be small even after it travels a long distance. In other words, the effective refractive index of the PC should be near zero (because $n \propto k$ ). Figure 1 shows that within the whole BZ, the eigenstates at the double Dirac point are the only Bloch states that can be excited by an externally incident wave at that frequency, which is relatively low $\left(\lambda_{0} \approx a / 0.61\right)$ so that diffraction of waves is avoided.
Therefore, in the frequency region around the double Dirac point, the PC should behave like a ZIM. In the following, we will study the wave transport behaviors associated with the double Dirac cone and reveal its connection to the ZIM. We put a slab of PC into a straight waveguide channel, as shown in Fig. 3(a), with sound hard boundary conditions on the upper and lower walls of the channel. The surface normal of the PC slab is along the $\Gamma M$ direction.

When a plane wave at a frequency below the double Dirac point is impinging on the PC slab from the left, the accumulated phase change of the wave inside the slab is easily observed, as illustrated in Fig. 3(a). However, when the frequency of the incident wave is very close to the double Dirac point, the phase change becomes almost invisible, as shown in Fig. 3(b), where only $40 \%$ of the incident wave energy transmits through the PC slab. Zero phase change implies that the refractive index is near zero; low transmission indicates that the impedance does not match the background. To enhance the transmission, we use a simple technique. By truncating the cylinders in the first/last row of the PC slab, we can effectively tune the surface impedance of the slab, and it will influence and determine, to a large extent, the total transmission coefficient of the PC slab. Figure 3(c) shows that total transmission is achieved, where

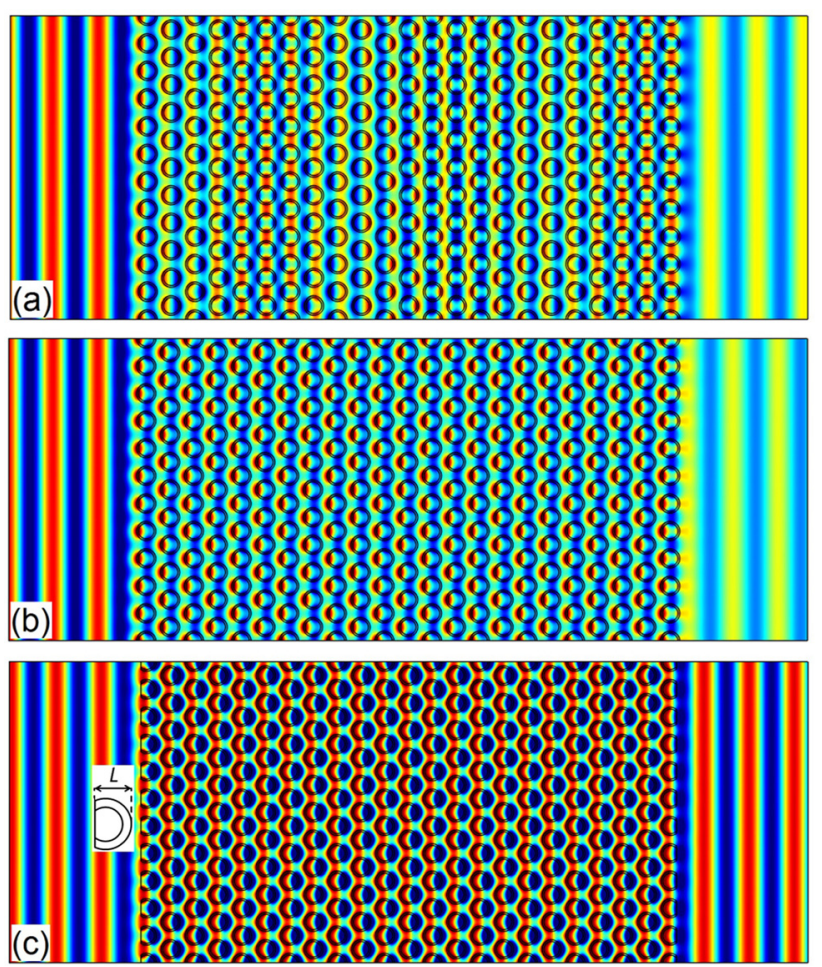

FIG. 3. (a) The pressure field distributions when a $\Gamma M$-direction plane wave is incident on a PC slab, whose structure is the same as that shown in Fig. 1(a). The PC slab is put inside a straight waveguide with sound hard boundary conditions on the upper and lower walls of the waveguide. The frequency of the incident wave is $\omega=0.5906\left(2 \pi c_{0} / a\right)$ below the double Dirac point frequency. Substantial phase change can be observed inside the PC slab. (b) The same as (a), except that the frequency of the incident wave is $\omega_{0}=0.6092\left(2 \pi c_{0} / a\right)$, exactly at the double Dirac point frequency. No phase change is observed, although only $40 \%$ of the incident wave transmits through the slab. (c) The same as (b), except that a straight-cut truncation is introduced at the two surfaces of the PC slab. The inset of (c) gives a schematic picture of the core-shell cylinders in the first row after truncation, with the cut parameter, $L=1.609 R_{1}$. 
the cutting parameter, $L$ (the height of the circular segment shown in Fig. 3(c)), is $1.609 R_{1}$.

To obtain the effective parameters of the PC slab, we adopted a standard retrieval method as follows. Assuming that the PC slab shown in Fig. 3(c) is replaced by a homogeneous material that gives the same scattering characteristics, the effective refractive index, $n_{\text {eff }}$, and the effective relative impedance, $Z_{r}$, of the PC slab can be retrieved from the complex transmission and reflection coefficients for a normally incident plane wave $\mathrm{w}^{34-37}$

$$
\begin{gathered}
Z_{r}=\frac{Z_{\text {eff }}}{Z_{0}}= \pm \sqrt{\frac{(1+r)^{2}-t^{2}}{(1-r)^{2}-t^{2}}}, \\
e^{i k_{e f f} d}=e^{i n_{e f f} k_{0} d}=\frac{t}{1-r \frac{Z_{r}-1}{Z_{r}+1}},
\end{gathered}
$$

where $Z_{0}\left(k_{0}\right)$ and $Z_{\text {eff }}\left(k_{\text {eff }}\right)$ are, respectively, the impedances (wave numbers) of water and the PC slab and $d$ is the equivalent thickness of the hypothetical continuous material. $t$ and $r$ represent the complex transmission and reflection coefficients, respectively. With a passive medium, the sign ambiguity of Eq. (3) can be eliminated by requiring that $\operatorname{Re}\left(Z_{r}\right) \geq 0$. Once $Z_{r}$ is known, $n_{\text {eff }}$ can be obtained according to Eq. (4). We note that the real part of $n_{\text {eff }}$ has multiple branches. The natural choice is to let $\left|\operatorname{Re}\left(n_{\text {eff }}\right)\right|$ or $\left|\operatorname{Re}\left(k_{\text {eff }}\right)\right|$ be as small as possible. ${ }^{31,38}$ On the other hand, the pressure field distributions shown in Fig. 3(c) indeed exhibit zero phase change inside the PC slab, which also suggests that $n_{\text {eff }}$ is very small or near zero.

Figure 4(a) shows the retrieved relative impedance, $Z_{r}$, as a function of the frequency, which is close to 2 except that near the double Dirac point frequency; it contains a Lorentzian-like feature. This Lorentzian-like artifact is caused by the close-to-one behavior of $t$ accompanied by the
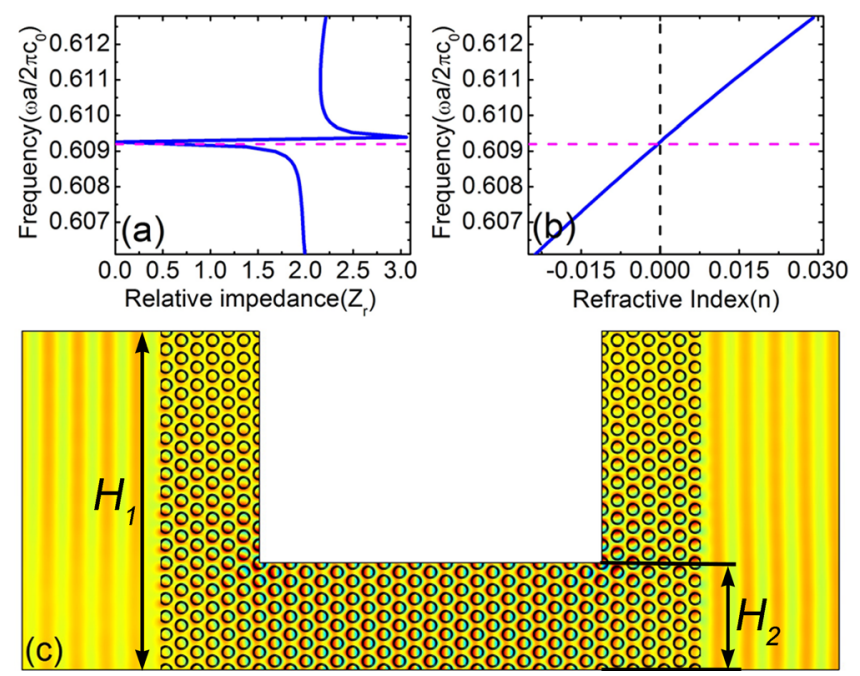

FIG. 4. (a) The relative impedance, $Z_{r}$, as a function of the frequency. (b) The effective refractive index, $n_{\text {eff }}$, as a function of the frequency. $n_{\text {eff }}$ is very close to zero around the double Dirac point frequency, which is marked with magenta dashed lines. (c) The pressure field distributions when a plane wave is impinging on a U-shaped narrow channel filled with our PC at the double Dirac point frequency. Sound hard boundary conditions are imposed on all walls of the channel. $H_{2} / H_{1} \approx 0.3$. The incident wave can transmit through the U-shaped narrow channel. close-to-zero behavior of $r$ in the vicinity of the double Dirac point frequency, as can be easily inferred from the right-hand side of Eq. (3), and this artifact is an inherent characteristic of the retrieval procedure, which was observed in Ref. 37. The effective refractive index, $n_{\text {eff }}$, demonstrated in Fig. 4(b), is very close to zero around the double Dirac point frequency, which coincides with our expectation and means that the PC slab can be effectively described as a ZIM. With a ZIM, one can achieve many interesting transport properties. In what follows, we demonstrate an anomalous tunneling effect achieved by our PC.

We construct a U-shaped waveguide channel by shrinking the middle part of the waveguide we used previously, at a ratio of $H_{2} / H_{1} \approx 0.3$. Sound hard boundary conditions are imposed on all the walls of the waveguide. At the double Dirac point frequency, the incident plane wave can totally transmit through the U-shaped channel with zero phase change inside the channel and then exit from the right-side surface as a plane wave with little phase distortion. We emphasize that this tunneling effect is conceptually different from the usual Fabry-Perot (FP) resonance. First, with a U-shaped narrow neck waveguide, tunneling and total transmission of wave energy can be realized at different neck lengths at the same frequency, while the FP resonant frequency is highly dependent on the horizontal length of the neck. Second, inside the narrow neck, substantial phase change can be observed for the FP resonance, e.g., the phase in the middle of the neck is usually different from that at the boundary of the neck. In our PC, on the contrary, no phase change is observed throughout the neck.

To conclude, we designed a 2D PC in which a double Dirac cone is realized at the center of the BZ. The PC consists of a triangular array of rubber-coated iron cylinders embedded in a water host, and the double Dirac cone is created by the accidental degeneracy of two double-degenerate eigenstates. The double Dirac cone contains two identical Dirac cones, whose linear slopes and isotropic features can be accurately predicted and verified by a first-principles perturbation method. The effective refractive index and relative impedance of a PC slab can be obtained by using a standard retrieval method, which shows the equivalence between the PC slab and an acoustic ZIM around the double Dirac point frequency. Total transmission of wave energy and zero phase change inside the PC slab are unambiguously demonstrated in two examples, and these phenomena are robust regardless of whether or not the shear wave modes inside the solid components are included. In addition to these features, our findings suggest diverse possibilities for potential applications in multiple fields such as cloaking of an object, directional emission, the Zitterbewegung effect associated with a fourfold degenerate state, ${ }^{22}$ and the phase matching effect on the enhancement of nonlinearity. ${ }^{32}$

The authors wish to thank Dr. Min Yang for helpful discussions. This research reported here was supported by the National Natural Science Foundation of China (Grant No.11274120), the Fundamental Research Funds for the Central Universities (Grant No. 2014ZG0032), and King Abdullah University of Science and Technology (KAUST). 
${ }^{1}$ A. H. Castro Neto, F. Guinea, N. M. R. Peres, K. S. Novoselov, and A. K. Geim, Rev. Mod. Phys. 81, 109 (2009).

${ }^{2}$ S. Tang and M. S. Dresselhaus, Nano Lett. 12, 2021 (2012).

${ }^{3}$ S. Tang and M. S. Dresselhaus, Nanoscale 4, 7786 (2012).

${ }^{4}$ R. Shen, L. B. Shao, B. Wang, and D. Y. Xing, Phys. Rev. B 81, 041410 (2010).

${ }^{5}$ R. A. Sepkhanov, Ya. B. Bazaliy, and C. W. J. Beenakker, Phys. Rev. A 75, 063813 (2007).

${ }^{6}$ F. D. M. Haldane and S. Raghu, Phys. Rev. Lett. 100, 013904 (2008).

${ }^{7}$ X. Zhang and Z. Liu, Phys. Rev. Lett. 101, 264303 (2008).

${ }^{8}$ X. Zhang, Phys. Rev. Lett. 100, 113903 (2008).

${ }^{9}$ S. R. Zandbergen and M. J. A. de Dood, Phys. Rev. Lett. 104, 043903 (2010).

${ }^{10}$ X. Huang, Y. Lai, Z. H. Hang, H. Zheng, and C. T. Chan, Nat. Mater. 10, 582 (2011).

${ }^{11}$ D. Torrent and J. Sánchez-Dehesa, Phys. Rev. Lett. 108, 174301 (2012).

${ }^{12}$ K. Sakoda, Opt. Express 20, 3898 (2012).

${ }^{13}$ K. Sakoda, Opt. Express 20, 25181 (2012).

${ }^{14}$ F. Liu, X. Huang, and C. T. Chan, Appl. Phys. Lett. 100, 071911 (2012).

${ }^{15}$ P. Peng, J. Mei, and Y. Wu, Phys. Rev. B. 86, 134304 (2012).

${ }^{16}$ L. Lu, L. Fu, J. D. Joannopoulos, and M. Soljačić, Nat. Photonics 7, 294 (2013).

${ }^{17}$ M. J. A. Smith, R. C. McPhedran, and M. H. Meylan, Waves Random Complex Media 24, 35 (2014).

${ }^{18}$ Y. Wu, Opt. Express 22, 1906 (2014).

${ }^{19}$ Y. Li, Y. Wu, X. Chen, and J. Mei, Opt. Express 21, 7699 (2013).

${ }^{20}$ J. Mei, Y. Wu, C. T. Chan, and Z.-Q. Zhang, Phys. Rev. B 86, 035141 (2012).

${ }^{21}$ K. Sakoda, Opt. Express 20, 9925 (2012).
${ }^{22}$ Z.-G. Chen, X. Ni, Y. Wu, C. He, X.-C. Sun, L.-Y. Zheng, M.-H. Lu, and Y.-F. Chen, Sci. Rep. 4, 4613 (2014).

${ }^{23}$ M. Silveirinha and N. Engheta, Phys. Rev. Lett. 97, 157403 (2006).

${ }^{24}$ S. Enoch, G. Tayeb, P. Sabouroux, N. Guérin, and P. Vincent, Phys. Rev. Lett. 89, 213902 (2002).

${ }^{25}$ J. Hao, W. Yan, and M. Qiu, Appl. Phys. Lett. 96, 101109 (2010).

${ }^{26}$ Q. Cheng, W. X. Jiang, and T. J. Cui, Appl. Phys. Lett. 99, 131913 (2011).

${ }^{27}$ V. C. Nguyen, L. Chen, and K. Halterman, Phys. Rev. Lett. 105, 233908 (2010).

${ }^{28}$ Z. Liang and J. Li, Phys. Rev. Lett. 108, 114301 (2012).

${ }^{29}$ R. Fleury and A. Alù, Phys. Rev. Lett. 111, 055501 (2013).

${ }^{30}$ P. Moitra, Y. Yang, Z. Anderson, I. I. Kravchenko, D. P. Briggs, and J. Valentine, Nat. Photonics 7, 791 (2013).

${ }^{31}$ S. Kocaman, M. S. Aras, P. Hsieh, J. F. McMillan, C. G. Biris, N. C. Panoiu, M. B. Yu, D. L. Kwong, A. Stein, and C. W. Wong, Nat. Photonics 5, 499 (2011).

${ }^{32}$ H. Suchowski, K. O'Brien, Z. J. Wong, A. Salandrino, X. Yin, and X. Zhang, Science 342, 1223 (2013).

${ }^{33}$ M. Kafesaki and E. N. Economou, Phys. Rev. B 60, 11993 (1999).

${ }^{34}$ D. R. Smith, S. Schultz, P. Markoš, and C. M. Soukoulis, Phys. Rev. B 65, 195104 (2002).

${ }^{35}$ X. Chen, T. M. Grzegorczyk, B.-I. Wu, J. Pacheco, Jr., and J. A. Kong, Phys. Rev. E 70, 016608 (2004).

${ }^{36}$ V. Fokin, M. Ambati, C. Sun, and X. Zhang, Phys. Rev. B 76, 144302 (2007).

${ }^{37}$ X.-X. Liu, D. A. Powell, and A. Alù, Phys. Rev. B 84, 235106 (2011).

${ }^{38}$ M. Yang, G. Ma, Y. Wu, Z. Yang, and P. Sheng, Phys. Rev. B 89, 064309 (2014). 